\title{
HUESO CON GRABADOS PALEOLÍTICOS DE LA COVA DE LES CENDRES (TEULADA, ALICANTE)
}

\author{
VALENTÍN VILLAVERDE BONILLA \\ Universidad de Valencia
}

\begin{abstract}
El hallazgo de un hueso decorado con una cierva en la Cova de les Cendres (Teulada, Alicante) permite constatar que la secuencia artística de la España meditarránea se completa hasta las últimas etapas del Paleolítico superior, de acuerdo con su propia evolución industrial, y replantear el tema de las relaciones entre el arte del Magdaleniense Superior-Final y el del Epipaleolítico Antiguo.

The finding of a carved bone with a female deer in the Cova de les Cendres (Teulada, Alicante) confirms that the artistic sequence in Mediterranean Spain goes as far as the last stages of the Upper Paleolithic, according to their own industrial evolution, and allows us to restate the topic of the relations between the art of the Later Upper Magdalenian and those of the Earlier Epipaleolithic.
\end{abstract}

La pieza que queremos dar a conocer en estas líneas procede del nivel V - campaña de 1974-de la Cova de les Cendres. Habiendo sido recientemente localizada por M. Pérez Ripoll al estudiar la fauna de dicha campaña.

En el mismo sector se hallaron en su día un arpón y otros materiales líticos de clara pertenencia al Paleolítico Superior, que pusieron de manifiesto la existencia de momentos correspondientes al Magdaleniense (LLOBREGAT ET ALII, 1981). La campaña de urgencia realizada en 1981, en la que se contempló la realización de un sondeo comprobatorio de tales indicios, confirmó estas previsiones y mostró la existencia de un rico e interesante Magdaleniense Superior que, tras la aparición de un nuevo arpón y una abundante industria lítica y ósea, quedó definido por la buena proporción de buriles y hojitas de borde abatido, siendo significativa la presencia de triángulos escalenos (VILLAVERDE, 1981).

Con posterioridad han continuado los trabajos arqueológicos en el yacimiento, centrándose la labor en los niveles Neolíticos (BERNABEU, 1986; VILLAVERDE y BERNABEU, 1986), reanudándose en el presente año la excavación de los paquetes paleolíticos y neolíticos. Fruto de la campaña de
1986 (julio-septiembre) ha sido la determinación de una mayor complejidad sedimentológica de la observada en 1981, especialmente en lo que hace referencia al contacto entre los niveles paleolíticos y neolíticos. Pudiéndose comprobar que el Neolítico antiguo supuso un proceso de erosión antrópica importante, con la ejecución de estructuras de variada tipología y utilidad, que en todos los casos afectaron, aunque de diferente manera e importancia, a alguno de los niveles que les precedían.

Ante la imposibilidad de adelantar resultados, dado que los trabajos están en curso y el estado inicial en el que se encuentra la excavación de los paquetes paleolíticos, bástenos indicar, en la medida en que sirve para explicar la problemática que acompaña la posición estratigráfica del objeto de arte mueble del que aquí tratamos, que los niveles de cerámica cardial que inician la secuencia Neolítica del yacimiento engloban habitualmente y dependiendo sobre todo de fenómenos de carácter zonal relacionados con las estructuras que en ellos se localizan, materiales de indudable adscripción al Paleolítico Superior-Final. Este fenómeno, teniendo en cuenta que en los sectores hasta ahora estudiados no aparecen materiales atribuibles al Epipalelítico geométrico, unido al hecho de que la mayor parte 
de las piezas que aparecen en los primeros niveles del Neolítico sean de tipología magdaleniense, parece indicar que los niveles del Epipaleolítico microlaminar si bien pueden estar presentes en el yacimiento deben ser poco potentes, ya sea por razones de orden sedimentológico, ya de orden puramente cultural.

Estas modificaciones, que amplían los resultados obtenidos en la campaña de 1981 y son fruto de la extensión de la excavación a una mayor superficie, no han significado, con todo, una variación en la determinación de los rasgos industriales que en su día se avanzaron sobre el Magdaleniense Superior, pues con independencia del enriquecimiento estratigráfico y tal vez industrial que pueda establecerse en el futuro, lo cierto es que el nivel entonces estudiado se ha visto confirmado a partir de los recientes trabajos, con la aparición de nuevas piezas óseas (azagayas de doble bisel, varillas, etc.) que amplían coherentemente su definición.

Por lo que respecta al hueso grabado que a continuación describimos, su aparición en el nivel V, en contexto Neolítico, se explica, teniendo en cuenta las consideraciones efectuadas, de la misma manera que en su día se hizo con los materiales finipaleolíticos que formaban parte del nivel IV.

\section{Descripción de la pieza}

Se trata de un fragmento, a su vez roto en tres partes, correspondiente a un metatarso izquierdo de Cervus elaphus, cuyas medidas son $11,4 \times 1,13 \times 0,71 \mathrm{cms}$. Sus limites coinciden con fracturas de aspecto reciente que, una vez orientado en base al tema decorativo que en él aparece, coinciden en su parte inferior con un trabajo antiguo de la pieza que parece corresponder a las facetas de preparación de una extracción para la fabricación de algún instrumento: un surco obtenido mediante incisión profunda repetida, que atraviesa casi la totalidad del espesor de la diáfisis, favoreciendo así su posterior fractura.

El tema central, y único que puede leerse en la actualidad, es la figuración, naturalista y estilizada, de un cérvido; más concretamente, la cabeza, arranque del pecho y línea cérvico-dorsal de una cierva. Esta representación aparece en compañía de otros trazos, en algunos casos claramente anteriores, que al ser parcialmente reutilizados complican ligeramente su dibujo.
Con independencia de las fracturas recientes, un examen detenido de los trazos en relación con el surco que limita la pieza por su lado inferior da a entender que este último se realizó con posterioridad al grabado del hueso.

En la ejecución de la cierva pueden observarse distintos tipos de trazos. Trazo múltiple, de surco en forma de $U$, en la línea del pecho; trazo repetido en la línea cérvico dorsal; trazo simple y somero, con surco en forma de $U$, en las líneas de la cabeza; y trazo simple y somero, superficial pero ancho, en las líneas correspondientes a las orejas. Los restan-

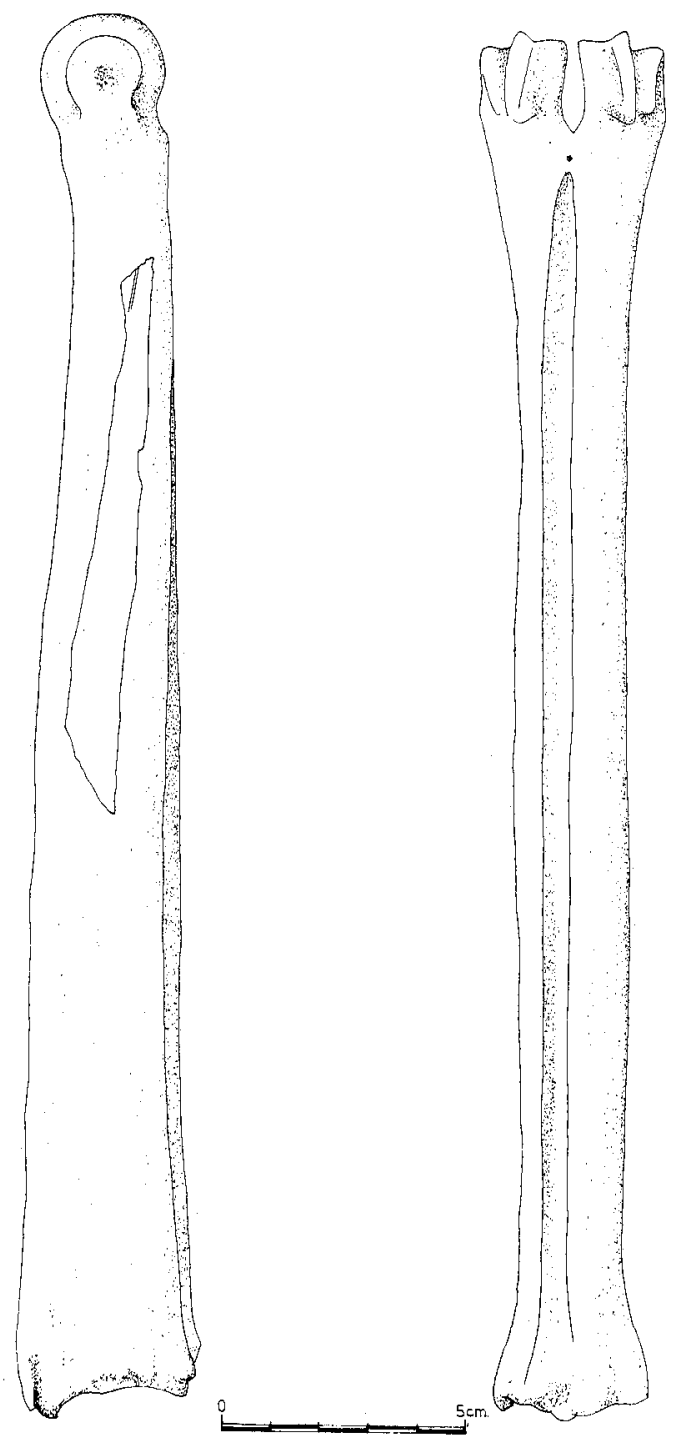

Fig. 1 Metatarso izquierdo de Cervus elaphus, con indicación de la situación del fragmento grabado. 

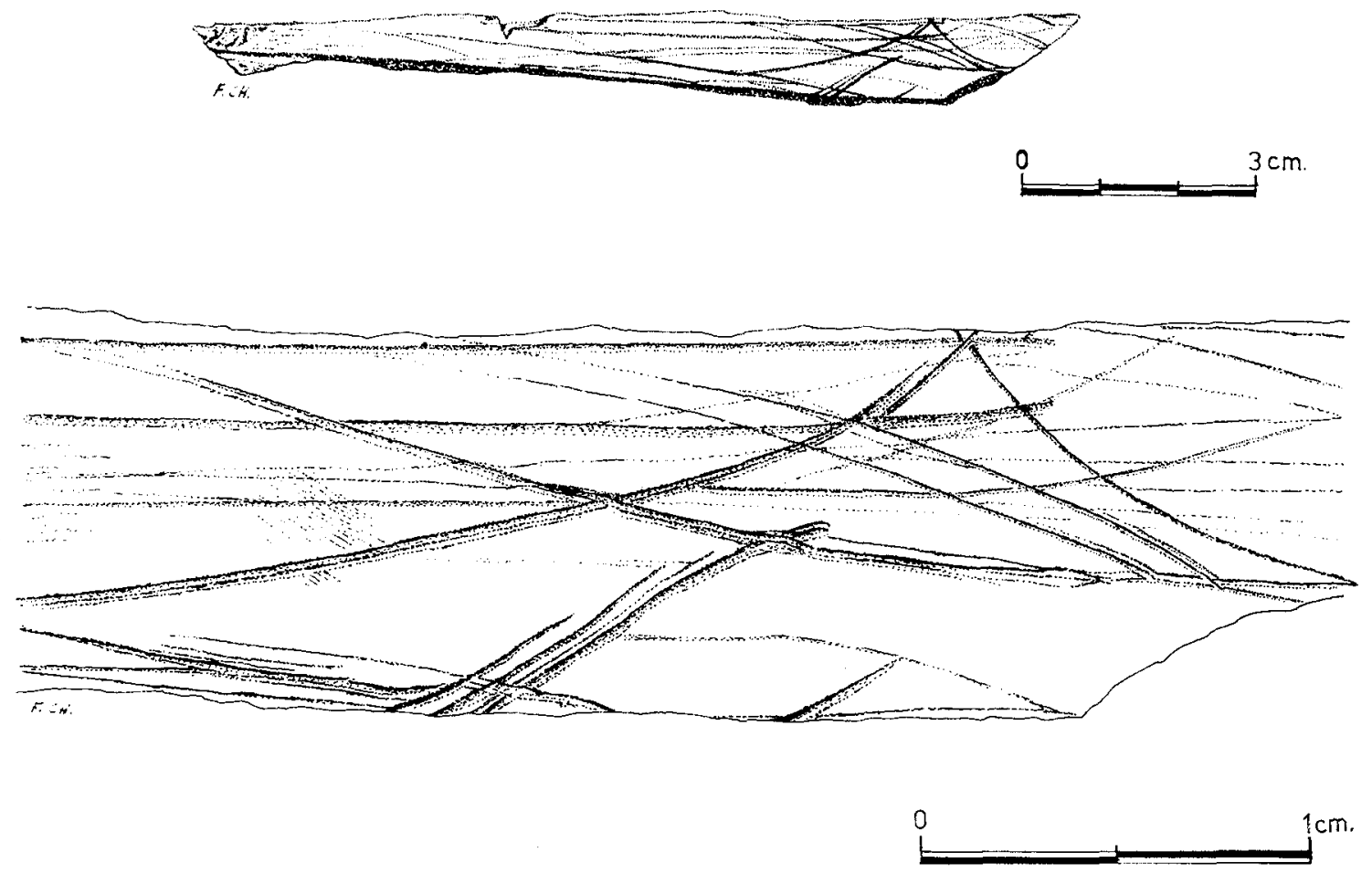

Fig. 2 Dibujo del hueso y detalle de la representación de la cierva.

tes trazos son simples y someros, con excepción de una línea larga y marcada que atraviesa la casi totalidad de la longitud de la pieza por su parte inferior, y en este caso aparecen combinados dos tipos de trazos, uno simple de sección en $\mathrm{V}$ y otro repetido.

Atendiendo a la composición o dibujo, el ritmo de ejecución que puede deducirse a partir del estudio de las superposiciones de los distintos trazos es el siguiente. En primer lugar se ejecutaron las líneas longitudinales que aparecen en la parte inferior del hueso y que, salvo en uno de los casos, no guardan relación directa con los trazos que conforman la figura de la cierva. Vienen después las líneas correspondientes al pecho y parte cérvico-dorsal, en el primer caso cortando las líneas anteriores y en el segundo partiendo de una de ellas, lo que produce un efecto de continuación o prolongación de la línea dorsal. Seguidamente las líneas de las orejas y de la quijada que, para finalizar, son en ambos casos cortadas por las dos líneas paralelas que conforman la frente del animal.

Resalta, al juzgar estilísticamente la representación, el esquematismo o estilización de la misma, acentuado por el hecho de que la mayoría de los trazos que se prolonguen exageradamente más allá del contorno. Es el caso de la oreja inferior, cuyo trazo proviene de la parte izquierda, extendiéndose por casi la totalidad de la superficie del hueso, y que al llegar a la línea cervical penetra en su surco desviándose de manera casi imperceptible hacia arriba. $\mathrm{O}$ de las tres líneas que conforman la cabeza, y en especial la de la mandíbula, que atraviesa el cuello sobrepasándolo y no termina en el morro.

Dado el carácter fragmentario de la pieza, nada puede precisarse sobre aspectos tales como el encuadre o la composición, a no ser el recordar la posible superposición temática que se deduce de la disposición de los restantes trazos que aparecen en relación con la figura de la cierva.

\section{Valoración en el contexto del Arte Paleolítico del Mediterráneo español}

Las consideraciones a realizar vienen limitadas por las condiciones de su hallazgo y su carácter fragmentario. Aspectos que inciden tanto en la valoración del tema en relación con el soporte, como en 


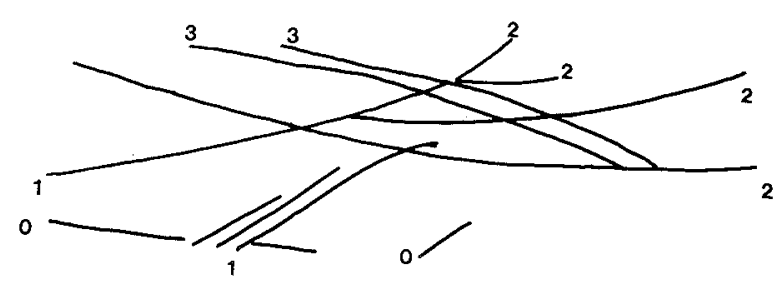

Fig. 3 Esquema de superposición de trazos.

su atribución a una fase cultural o cronológica precisa.

Su ámbito de comparación inmediato viene dado por la colección de plaquetas grabadas y pintadas de la Cova del Parpalló (Gandía, Valencia), donde se han basado gran parte de los conceptos que informan la sistematización del Arte Paleolítico de la España mediterránea (Pericot, 1942; Jordá, 1978 y 1985; Fortea, 1978). La estilización que en variadas ocasiones se ha asociado a su producción y que en efecto constituye, salvo en contados casos, uno de sus rasgos más característicos, coincide con el planteamiento de la pieza de Cendres. Y muy especialmente si consideramos alguna de las representaciones de cérvidos de sus capas superiores, donde se observan idénticas concepciones en la proyección de cabeza y cuello hacía adelante y en la forma de representar las orejas en $\mathrm{V}$. Nótese al respecto que coincidiendo con el Solútreo-gravetiense se produce en Parpalló una nueva forma de representar estos animales, y así el dibujo típico durante el Solutrense - las cabezas realizadas mediante tres trazos, de los que dos se abren en abanico, uno para dibujar la línea de la mandíbula y el otro una de las orejas, mientras que el tercero a la vez que cierra el espacio, figurando la frente, se prolonga hacia atrás, conformadno la segunda oreja (FORTEA, 1978)va a dar paso a partir de Solútreo-gravetiense $y$ durante las etapas siguientes, a la representación de cabezas más perfiladas, de trazos que se cierran y cuidan más el acabado del morro, todo ello incorporando una nueva forma de tratar las orejas, que se resuelven mediante dos trazos en forma de $\mathrm{V}$, o formas incluso más naturalistas que sustituyen la perspectiva biangular recta por la triangular (VILLAVERDE Y MARTÍ, 1984). Estas últimas sobre todo en los niveles Magdalenienses, si bien en compañía de las más esquemáticas hasta las últimas capas del yacimiento (véase la plaqueta de 0-0, $5 \mathrm{~m}$., fig. 471, en PERICOT, 1942).
Sin embargo, y sin quitar importancia a la valoración estilística de la pieza de Cendres en su comparación con los datos que se deducen de Parpalló, lo que realmente nos parece importante al tratar de esta pieza es el como incide, junto a otros hallazgos recientes, en la confirmación de que el fenómeno circunscrito a uno o dos yacimientos «especiales»; y lo que es más importante, la constatación de que la secuencia artística de esta región se completa hasta las últimas etapas del Paleolítico Superior, de acuerdo con su propia evolución industrial y favoreciendo de esta manera el replanteamiento de un tema tan interesante como el de las relaciones entre el arte del Magdaleniense Superior-Final y el del Epipaleolítico Antiguo o Inicial. Problemática ésta que hace pocos años se veía condicionada por la inexistencia de niveles encuadrables con rotundidad en el Magdaleniense Superior.

Los datos se han multiplicado en apenas unos años y el actual registro arqueológico, si bien provisiolnal en muchos aspectos, permite retomar incluso la problemática de los tramos superiores de Parpalló, considerando la posibilidad de que también en este yacimiento pueda contemplarse la existencia de momentos correspondientes a esta fase del Magdaleniense.

La publicación en 1942 de la secuencia industrial y artística de Parpalló partió, refiriéndonos al Magdaleniense, de la idea de que su inicio y final coincidían grosso modo con los denominados Magdalenienses I y IV. La industria ósea, y más específicamente las azagayas con monobisel en lengüeta y decoración en espiga y los «protoarpones», constituyeron los elementos sobre los que se apoyó esta visión. Esta ordenación, responsable de que a lo largo de muchos años se haya venido considerando al Magdaleniense Superior como una etapa inexistente en la región central mediterránea, ha venido siendo criticada en los últimos años en ciertos sectores bibliográficos (FORTEA ET ALII, 1983; JORDÁ, 1986). En ello han intervenido de manera determinante la constatación de la existencia de Magdaleniense Superior tanto en Cendres como en el Tossal de la Roca (Vall d'Alcala, Alicante) y Matutano (Vilafamés, Castellón), como la aportación que la industria ósea de este último yacimiento ha sumado a una discusión hasta ahora sólo planteable a partir de la industria lítica y la evolución estilística de las plaquetas de Parpalló. Así, la convivencia en el estrato III de Matutano de arpones de dientes bien destacados con otros de morfología 

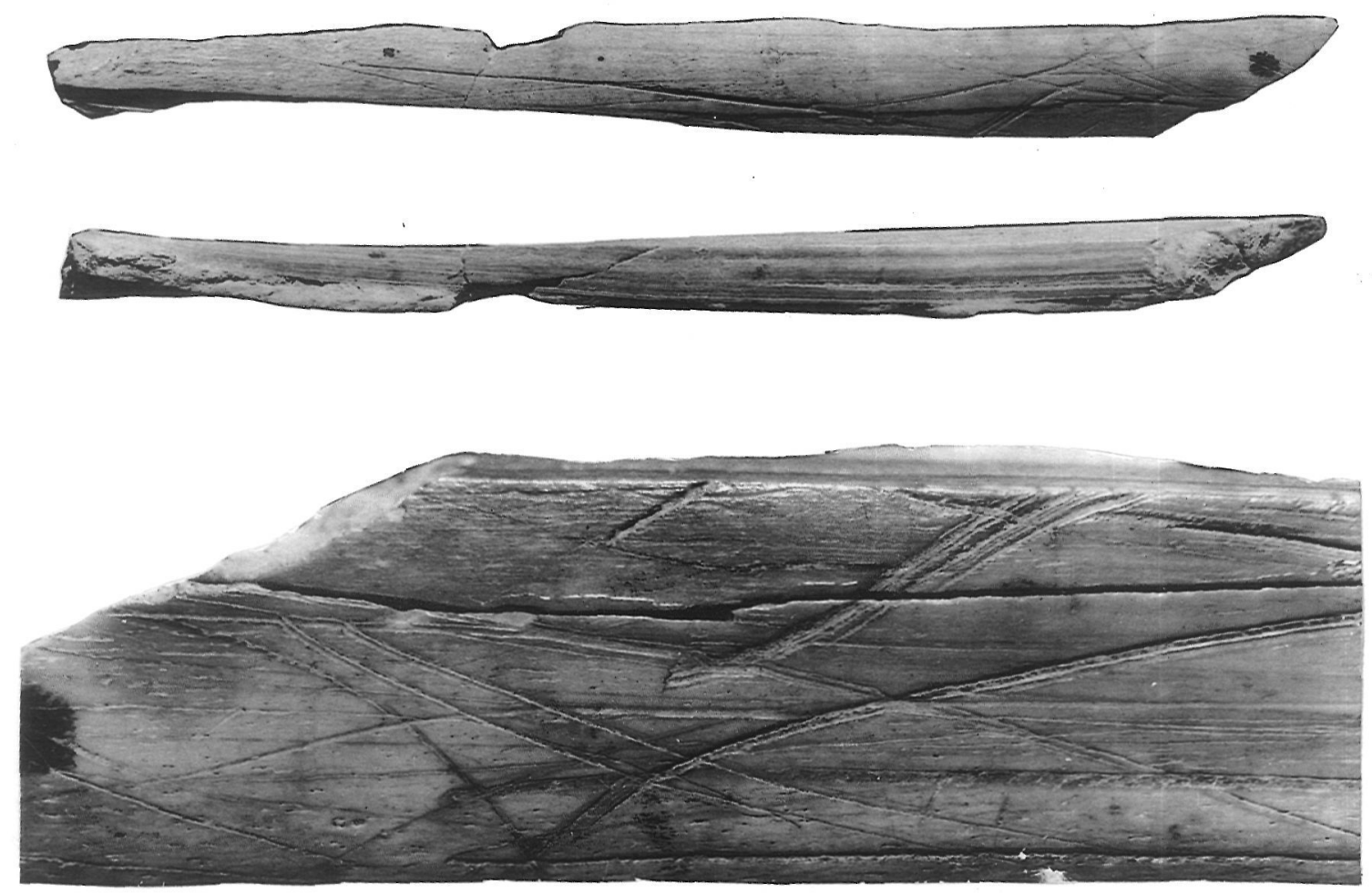

Fig. 4 Cendres. Foto ampliada del hueso grabado y detalle muy aumentado de la cierva. Foto Gil-Carles.

semejante a los de Parpalló (OLARIA ET ALII, 1981; fig. 19.1 y 18.7), permite retomar el límite cronológico atribuido a los arpones de Parpalló; circunstancia que enlazaría en otro orden de discusión, coñ la presencia de triángulos escalenos, piezas cuyos paralelos conducen de nuevo no sólo al Magdaleniense Superior de Cendres (VILLAVERDE, 1981), sino al conjunto del Magdaleniense Superior mediterráneo, desde Gerona a Málaga (FORTEA, 1973).

La posibilidad de que Parpalló incluya, por tanto, en su secuencia niveles posteriores al Magdaleniense Medio, a la vez que hace más coherente su propia evolución, pues acota el ámbito cronológico e industrial de un Magdaleniense Medio que aparecía, en su comparación con otras regiones, claramente desbordado, facilita una nueva óptica con la que abordar el análisis de su ciclo artístico, discordante en sus últimos tramos con la idea de que su límite debía situarse en el denominado estilo IV antiguo de Leroi-Gourhan. Recordemos ahora, sin ánimo de tomar exhaustivamente un tema que merece un mayor estudio, el realismo, detalle y logro de proporciones de alguna de las plaquetas con representaciones zoomorfas del tramo IV (FORTEA, 1978), o la existencia de convenciones de pelaje o modelado que nos llevarían paralelos cantábricos avanzados (VILLA VERDE, en prensa), o la proliferación de signos complejos que caracteriza la producción artística de las últimas capas del yacimiento.

Por otra parte, esta posibilidad no entra en contradicción con los datos que poseemos de los restantes yacimientos del País Valenciano, que por cronología y con independencia de la exacta atribución cultural de sus niveles, proporcionan piezas de arte mueble que son claramente encuadrables en un genérico Paleolítico Superior Final.

Las piezas de Cendres, el Tossal de la Roca (APARICIO y SAN VALERO, 1983), o Matutano (OLARIA ET ALII, 1981), parecen enlazar así con las de los niveles superiores de Parpalló, configurando un ciclo artístico de evidente amplitud geográfica y claras coincidencias con otras regiones. 
Quedaría tan sólo, por ahora, como algo específico de Cendres la naturaleza ósea del soporte, predominantemente lítico a juzgar por los datos disponibles, pues se añadirían tan sólo un hueso grabado, con un équido y un cérvido, del Solútreo-gravetiense (APARICIO, 1981); un cáprido, sobre punta larga de extremo aplanado, y otro cáprido sobre punta aplanada, del Magdaleniense Inicial; y una representación de pez, del tramo III del Magdaleniense de Parpalló, probablemente del Magdaleniense Medio. Y frente a estas piezas encontramos, además de las de Parpalló y refiriéndonos siempre a los temas zoomorfos naturalistas, con el ya mencionado hallazgo de Matutano - un canto grabado por ambas caras con representaciones de cérvidos-y del Tossal de la Roca - otro canto con grabados de un cáprido y un posible cérvido-, datos a los que habría que añadir las noticias, aún no publicadas, de plaquetas grabadas en la Cova del Barranc de l'Infern (Fleix) y otras piezas de Matutano.

Sin embargo, no parece aconsejable extraer conclusiones de esta cuestión, especialmente si consideramos el incremento de los trabajos de campo en yacimientos de cronología finipaleolítica.

Donde si parece oportuno centrar la atención es en la pieza publicada de Matutano, única de las anteriormente citadas que es posible encuadrar estratigráficamente, ya que su discusión incide directamente en la valoración del final del ciclo artístico Magdaleniense y su relación con el escaso, pero interesante, arte mueble del Epipaleolítico microlaminar, representado por la cierva grabada sobre plaqueta de pizarra del nivel II de Sant Gregori de Falset (VILASECA, 1934; FORTEA, 1973).

El canto grabado de Matutano pertenece al nivel IB del sondeo de 1979, caracterizado en lo lítico por la elevada proporción de raspadores $(22,6 \%)$, lo exiguo del índice de buril $(6 \%)$, y la poca entidad del utillaje microlaminar retocado. Nivel calificado de Magdaleniense Superior a partir del resultado de la fecha de C-14 obtenida a $1,74 \mathrm{~m}$. de potencia: 12.090 \pm 170 B. P. (OLARIA ET ALII, 1981).

De seguir estas indicaciones nos encontraríamos, por tanto, con una pieza que se encuadraría dentro del Magdaleniense Superior-Final, cerrando un ciclo artístico que, por cronología, no quedaría excesivamente alejado de la plaqueta de Sant Gregori, para la que, a su vez, cabría pensar en momentos próximos a los que fechan en el nivel VI de la
Cata Oeste-1970 de Mallaetes, con una cronología de 10.370 \pm 105 B. P. En un contexto industrial que corresponde a las primeras fases del Epipaleolítico microlaminar (FORTEA, 1973; FORTEA y JORDÁ, 1976).

Sin embargo, varios aspectos concurren en la secuencia propuesta para Matutano que nos parecen susceptibles de ser interpretados de manera distinta a como lo han hecho los excavadores del yacimiento. En primer lugar, la escasa diferencia cronológica existente entre los distintos estratos o niveles de su secuencia, especialmente en lo que hace referencia a los niveles IB, IIB y III, fechados respectivamente en $12.090 \pm 170,12.390 \pm 190$ y $12.130 \pm 180 \mathrm{~B}$. P. Esto es, cronologías que prácticamente se solapan a pesar de las diferencias de profundidad que separan las muestras. $Y$ en segundo lugar, todo ello en relación con una evolución industrial que se caracteriza no por la uniformidad, sino por la existencia de importantes transformaciones, en especial si centramos la atención en la comparación de los estratos III y IB. Aquél con una rica y variada industria ósea, con arpones y azagayas, y una composición del utillaje lítico presidida por la buena proporción de buriles $(26 \%)$ con relación a los raspadores $(16 \%)$; mientras que el nivel IB, sin apenas industria ósea, se caracteriza por lo contrario. Como se ha señalado, estas diferencias se inscriben en un claro proceso de transformación industrial, perfectamente observable a lo largo de los niveles IIA, IIB y IIC, que podría resumirse por el progresivo descenso del índice de buril, neto a partir del momento en el que la industria ósea decae en su importancia - el nivel IIC-, y un aumento del índice de truncaduras, todo acompañado de un índice de raspadores que aunque aumenta lo hace rápidamente para estabilizarse casi después, si bien con tendencia a la disminución.

Sin detenernos en el caso específico de las truncaduras, que en sí mismas no permiten mayores comparaciones, lo que si puede señalarse es que la dinámica que preside la evolución de Matutano no se aleja mucho de la que en principio puede establecerse para el proceso Magdaleniense SuperiorFinal y Epipaleolítico microlaminar. Este último vinculado en sus primeros momentos, tanto por la talla, la tipometría o la tipología, a las industrias Magdalenienses que le preceden. Tal y como se deduce de la comparación de los niveles Epipaleolíticos de Mallaetes, Sant Gregori (FORTEA, 1973) o, incluso, Ambrosio (SUÁREZ, 1981), con los de Cendres, los 
niveles inferiores de Matutano y el Tossal de la Roca, $\mathrm{u}$ otros yacimientos finipaleolíticos más septentrionales, como sería el caso de Coma d'Infern (SOLER, 1980) o el Cingle Vermell (VILA ET ALII, 1986).

Esta idea de vinculación, recogida recientemente en algunos trabajos que tienden a considerar las primeras fases del Epipaleolítico como un verdadero Epimagdaleniense (FORTEA ET ALII, 1983; FORTEA, 1985 y 1986), no ocultaría, con todo, la existencia durante el Epipaleolítico de un importante proceso de regionalización. Proceso de regionalización que en el ámbito mediterráneo, a juzgar por las distintas variantes industriales existentes en los yacimientos mencionados, ampliaría la que en su momento ofreció la completa síntesis que sobre el período realizó Fortea al distinguir dentro del complejo microlaminar las variantes tipo Sant Gregori y Mallaetes, y en la que el caso mismo de Matutano, menos entroncado con lo microlaminar en el utillaje retocado, podría constituir quizás una variante a sumar a la también peculiar industria de Coma d'Infern.

De seguir esta interpretación el nivel IB de Matutano, y con ello volvemos a las implicaciones que la cronología de su pieza de arte mueble posee en la valoración del ciclo artístico finipaleolítico, quedaría encuadrado en momentos posteriores al Magdaleniense, y por tanto la relación de su pieza con la plaqueta de Sant Gregori sería aun más estrecha que la manejada líneas arriba.

Que duda cabe, sin embargo, de que esta cuestión sólo podrá resolverse de manera definitiva con el recurso a los datos cronoestratigráficos $\mathrm{y}$ paleoambientales, favoreciendo así una visión cronológica que no descanse exclusivamente en la valoración de los procesos industriales. Por otra parte, tampoco puede excluirse por principio la posibilidad de que sea el mismo Magdaleniense Superior al que se diversifique, tal y como en cierto modo el nivel III de Matutano evidencia, al mostrarnos una variante menos rica en hojitas retocadas. $Y$ aquí, más que nunca, es necesario admitir que son importantes los vacíos cronológicos e industriales que jalonan el conocimiento del Paleolítico Superior Final y el Epipaleolítico del Mediterráneo peninsular; pero de lo que no cabe duda es de que una y otra fase mantienen una clara relación genética, ampliable sin dificultad al campo artístico, si bien sobre la base de un importante descenso de la producción y, lo más seguro, de su significación. Algo común, a fin de cuentas, con lo que en esos mismos momentos ocurre en otras regiones próximas.

\section{BIBLIOGRAFÍA}

APARICIO, J., 1981: «Nueva pieza de arte mobiliar parpallense», A. P. L., XVI, 39-58.

APARICIO, J. y SAN VALERO, J. 1983: El Primer Arte Valenciano. I Arte Parpallonés. Valencia.

BERNABEU, J., 1986: La Evolución del Neolítico en la Zona Oriental de la Península Ibérica. La Tradición Cultural de las Cerámicas Impresas. Tesis Doctoral inédita, Valencia.

LLOBREGAT, E.; MARTÍ, B.; BERNABEU, J.; VILLAVERDE, V.; GALLART, M. D.; PÉREZ, M.; ACUÑA, J. D. y ROBLES, F., «Cova de les Cendres (Teulada, Alicante)», 1981: Revista del Instituto de Estudios Alicantinos, n. ${ }^{\circ} 34,87-117$.

FORTEA, J., 1973: Los Complejos Microlaminares y Geométricos del Epipaleolítico Mediterráneo Español, Memorias del Seminario de Prehistoria y Arqueología, n. ${ }^{\circ}$, Salamanca.

FORTEA, J., 1978: «Arte Paleolítico del Mediterráneo Español», Trabajos de Prehistoria, 35.

FORTEA, J., 1985: «El Paleolítico y Epipaleolítico en la Región Central del Mediterráneo Peninsular. Estado de la cuestión industrial», en Arqueologia del País Valenciano: Panorama $y$ perspectivas, Alicante, 31-51.

FORTEA, J., 1986: «El Paleolítico Superior y Epipaleolítico en Andalucía. Estado de la cuestión cincuenta años después», en Actas del Congreso Homenaje a Luis Siret, 67-78.

FORTEA, J.; FULLOLA, J. M. ${ }^{a}$; VILLAVERDE, V.; DAVIDSON, I.; DUPRÉ, M. y FUMANAL, M. ${ }^{\text {a }}$ P., 1983: "Schéma peléoclimatique, faunique et chronostratigraphique des industries à bord abattu de la région méditerranéenne espagnole», Rivista di Scienze Preistoriche, vol. XXXVIII, 22-67.

FORTEA, J. y JORDÁ, F., 1976: «La cueva de Les Mallaetes y los problemas del Paleolítico Superior del Mediterráneo Español», Zephyrus, XXVIII-XXIX, 129-166.

JORDÁ, F., 1978: «Arte de la Edad de la Piedra», en Jordá, F. y Blázquez, J. M. ${ }^{a}$, Historia del Arte Hispánico, I. La Antigüedad, Madrid.

JORDÁ, F., 1985: «El Arte Prehistórico de la Región Valenciana», en Arqueología del País Valenciano: Panorama y perspectivas, 121-140, Alicante.

JORDÁ, F., 1986: «Paleolítico», en Jordá, F.; Pellicer, M.; Acosta, P. y Almagro, M., Historia de España. Prehistoria, Madrid.

OLARIA, C.; GUSI, F.; ESTÉVEZ, J.; CASABO, J. y ROVIRA, M. L., 1981: «El yacimiento magdaleniense superior de Cova Matutano (Villafanés, Castellón)». Estudio del Sondeo estratigráfico 1979, Cuadernos de Prehistoria y Arqueología Castellonenses, 8, 21-100.

PERICOT, L., 1942: La Cueva del Parpalló (Gandía). Madrid. SOLER, N., 1980: «El jaciment prehistòric de Coma d'Infern a les Encies (Les Planes, Girona)», Cypsela, III, 31-65.

SUÁREZ, A., 1981: «Cueva Ambrosio (Vélez Blanco, Almería). Nuevas aportaciones al estudio del Epipaleolítico del Sudeste peninsular», Antropología y Paleoecología Humana, 2, 43-53.

VILA, A., 1986: El Cingle Vermell: Assentament de caçadors del Xè. Mil.lenni B. P., Excavacions Arqueològiques a Catalunya, 5, Barcelona. 
VILASECA, S., 1934: «L'estació taller de silex de St. Gregori», Memoria de la Academia de Ciencias y Artes, vol. 23, 415-439.

VILLAVERDE, V., 1981: «El magdaleniense de la Cova de les Cendres (Teulada, Alicante y su aportación al conocimiento del magdaleniense mediterráneo peninsular», $P L A V$ Saguntum, 16, 9-35.

VILLAVERDE, V.; VELASCO, A.; ARIAS, J. M. y PORTELL, E., (n. p.): «Algunas precisiones sobre la técnica del grabado estriado en la Cova del Parpalló (Gandía, Valencia)», Saitabi.

VILLAVERDE, V. y BERNABEU, J., 1986: «Cova de les Cendres, en Arqueología en Alicante 1976-1986», Alicante, 51-52.

VILLAVERDE, V. y MARTÍ, B., 1984: Paleolitic i Epipaleolitic. Las societats caçadores de la Prehistoria valenciana, Valencia. 